\title{
RECRYSTALLIZATION OF AMORPHOUS LAYER IN ION IMPLANTED GaAs - TRANSMISSION ELECTRON MICROSCOPY STUDIES
}

\author{
J. JASIŃSKI \\ Institute of Experimental Physics, Warsaw University, Hoża 69, 00-681 Warsaw, Poland \\ AND Z. LILIENTAL-WEBER
}

Lawrence Berkeley National Laboratory, 1 Cyclotron Rd, Berkeley, CA 94720, USA

\begin{abstract}
$2 \mathrm{MeV}$ arsenic or gallium ions were used to produce nonstoichiometric buried amorphous layers in gallium arsenide. The mechanism of thermally induced regrowth of these layers was investigated using transmission electron microscopy. Low-temperature annealing resulted in nucleation of high densities of stacking faults. This was associated with the local nonstoichiometry of the amorphous layers. After annealing at high temperatures, in arsenic as well as in gallium implanted samples, two layers of voids, formed in result of vacancies clustering, were found in areas adjacent to the initial location of the amorphous-crystalline interfaces. A qualitative model of the formation of such layers was proposed.
\end{abstract}

PACS numbers: $61.80 . \mathrm{Jh}, 61.72 . \mathrm{Cc}, 61.72 . \mathrm{Ff}$

Ion implantation is a commonly used technique for electronic device processing. Therefore, in the past decades there have been numerous studies concerning the damage produced by ion implantation into semiconductors and its thermal recovery. Despite all these investigations there are still many unsolved problems. In this paper, two issues will be discussed. The first concerns the influence of local nonstoichiometry of an amorphous layer on its regrowth [1,2]. The second is the mechanism of formation of layers of voids, during high-temperature annealing.

Scmi-insulating [100] gallium arsenide (GaAs) wafers were implanted with $2 \mathrm{MeV}$ arsenic (As) or gallium (Ga) ions at doses of $1 \times 10^{16} \mathrm{~cm}^{-2}$ and $5 \times 10^{15} \mathrm{~cm}^{-2}$, respectively. For both the matcrials cross-sectional transmission electron microscopy (TEM) showed that applied doses were high enough to produce buried amorphous layers. After implantation some pieces of the materials were annealed for 20 minutes in selected temperatures between $300^{\circ} \mathrm{C}$ and $800^{\circ} \mathrm{C}$. In order to prevent arsenic outdiffusion from the surface annealing was performed under arsine ambient in MOCVD reactor. Cross-sectional TEM samples were prepared afterwards. 
TEM studies of annealed samples showed recrystallization of the amorphous layers, initiating at both amorphous-crystalline (a-c) interfaces. However, these studies also revealed that after annealing at low temperature $\left(300^{\circ} \mathrm{C}\right)$ a high density of stacking faults (SFs) and microtwins were present in recrystallized areas. SFs were found to nucleate in As as well as in Ga implanted samples. Asymmetry in their distribution was observed in both cases. The number of SFs in the deeper recrystallized part, formed at lower a-c interface, was significantly higher than that found in the part which had recrystallized at upper interface (closer to the surface). The appearance of high density of SF and described asymmetry of their distribution after low-temperature annealing can be understood in terms of local deviations from stoichiometric compositions in the amorphous phase. The following model is proposed for the mechanism of SF nucleation. When the implanted wafer is annealed, its amorphous layer recrystallizes at both upper and lower a-c interfaces, with a tendency to form stoichiometric material. This means that any local excess of $\mathrm{As} / \mathrm{Ga}$ atoms tends to be rejected into the amorphous phase during migration of the a-c interface. However, this process requires diffusion of excess As/Ga into the remaining amorphous material. For low annealing temperature an As/Ga diffusion rate is not fast enough, therefore, some of excess atoms can probably form small clusters at the interface. These clusters may act as SF nucleation centers. A more detailed description of this mechanism in the case of As implantation and an atomic model of the SF formation induced by small clusters of excess As has been presented elsewhere [3]. Based on this mechanism the observed asymmetry of SF densities can be understood. TRIM'92 [4] simulations when compared with TEM results of as-implanted samples showed that As/Ga excess depth profile was asymmetrically located within the amorphous layer. Concentration of the excess element, especially in the case of $A$ s implantation present near the lower a-c interface, was much higher than that near the upper interface [3]. This could explain the observed higher SF concentration in the lower part of the recrystallized layer after low-temperature annealing.

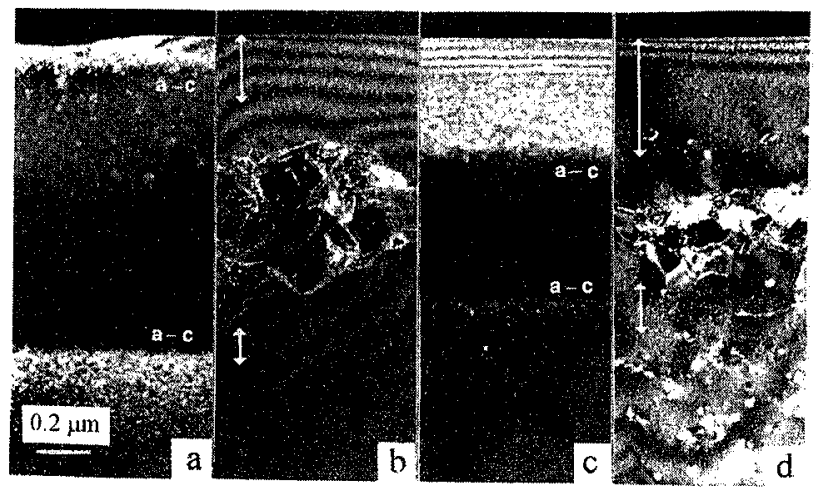

Fig. 1. TEM dark field micrographs of As and Ga implanted samples before (a), (c) and after (b), (d) high temperature annealing. For annealed samples, notice the presence of layers of voids in areas adjacent to the location of a-c interfaces in as-implanted samples. 
The implied assumption that an amorphous GaAs layer with little or no stoichiometry deviation can regrow forming a low SF density is in agreement with our recent unpublished TEM and RBS (Rutherford backscattering) studies of GaAs co-implanted with $200 \mathrm{keV}$ As and $185 \mathrm{keV} \mathrm{Ga}$ ions so as to preserve crystal stoichiometry. These studies have shown that after annealing $\left(\right.$ at $250^{\circ} \mathrm{C}$ ) the $\mathrm{SF}$ density in the co-implanted sample was much lower than those in samples implanted with only one element (As or Ga).

The second question concerns the mechanism of formation of voids in samples annealed at higher temperatures. In these samples, implantei with As as well as with $\mathrm{Ga}$ ions, two layers of small spherical defects, identified as voids, were found. In both cases the location of these layers corresponded to the location of the a-c interfaces in as-impianted samples (Fig. 1a, 1b). Similar results were also obtained for other implanted GaAs samples. Layer of voids was formed after high-temperature annealing when an amorphous layer was present in as-implanted material. Also in literature, an appearance of such layers of voids has been reported $[5,6]$. The mechanism of their formation involves diffusion of vacancies which are produced during implantation. In the following a qualitative model for the formation mechanism, of such layers of voids, will be proposed.

In general, accelerated ions implanted into a crystalline material lose their energy due to electronic and nuclear stopping. Only nuclear stopping displaces target lattice atoms and produces Frankel pairs (interstitials and vacancies). The ratio between electronic and nuclear stopping for incoming ion changes on the way from the surface down to the material. Initially, electronic stopping dominates in the whole energy loss process. When the ion slows down nuclear stopping increases and eventually starts to dominate. Therefore, the degree of radiation damage has a maximum value at some characteristic depth dependent on a target material, implanted ion and implantation energy. A consequence of such behaviour of a damage profile is possibility of buried amorphous layer creation when implantation dose is high enough. To explain the formation of amorphous phase, produced by ion implantation, the idea of threshold damage density (TDD) is used. The idea is based on the assumption that a crystal transforms into an amorphous material when local density of damage (in other words the concentration of produced vacancies) is higher than TDD. Therefore the amorphous material is first formed at the damage peak and then expands into two directions as the implantation dose increases. Schematic drawings of damage profile (profile of produced vacancies) and the location of TDD in case of (1) a buried amorphous layer and (2) an amorphous layer at the surface are shown in Fig. 2a and Fig. 2b, respectively.

We explain the formation mechanism of the layers of voids by the presence of significant concentration of vacancies in areas adjacent to the a-c interfaces. From the schematic pictures (Fig. 2) one can see that indeed in all these areas (see shaded regions) the concentrations of vacancies are only slightly. lower than that corresponding to the TDD. These vacancies are "trapped" in crystalline material. During thermal annealing they diffuse and form bigger clusters, that means voids. The higher the annealing temperature, the more effective vacancies diffusion. This should lead to an increase in void size and broadening of the layer with an increase in annealing temperature. 

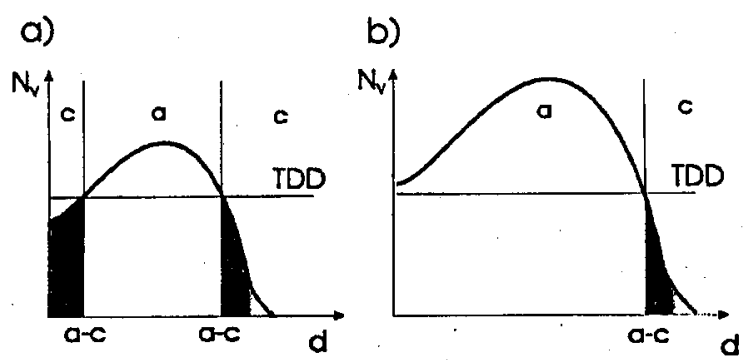

Fig. 2. Schematic drawings of a damage profile (profile of produced vacancies) and the location of TDD in case of (a) a buried amorphous layer and (b) an amorphous layer at the surface. Shaded regions represent crystalline parts of the material with a high density of vacancies, created during implantation. During annealing at high temperature these vacancies diffuse and form, observed in TEM, voids.

This model should apply not only for a buried amorphous layer (Fig. 2a) but also in the case of an amorphous layer at the surface (Fig. 2b).

In summary, we used TEM technique to study recrystallization of the buried amorphous $\mathrm{GaAs}$ layers produced by $\mathrm{MeV} \mathrm{As}$ or $\mathrm{Ga}$ ion implantation. It was postulated that local nonstoichiometry of the amorphous layer can result in the formation of high density of SF during low-temperature regrowth. After high-temperature annealing the layer of voids was observed in crystalline areas adjacent to the initial a-c interfaces. Proposed in this paper mechanism of formation of such layers suggests that the problem is more general and should be considered for different implanted crystalline materials, not only for GaAs.

This work was partially supported by AFOSR-ISSA-90-0009. The authors would like to thank Dr. C. Jagadish from Australian National University for $2 \mathrm{MeV}$ implantation and annealing of the samples.

\section{References}

[1] J.S. Williams, M.W. Austin, Appl. Phys. Lett. 36, 994 (1980).

[2] W.G. Opyd; J.F Gibbons, J.C. Bravman, M.A. Parker, Appl. Phys. Lett. 49, 974 (1986).

[3] J. Jasiński, Y. Chen, J. Washburn, H.FI. Tan, C. Jagadish, M. Kamińska, Appl. Phys. Lett. 68, 1501 (1996).

[4] J.F. Ziegler, J.P. Biersack, U. Littmark, The Stopping and Range of Ions in Solids, Pergamon, New York 1985.

[5] Kei-Yu Ko, Samuel Chen, S.-Tong Lee, G. Braunstein, Appl. Phys. Lett. 60, 1223 (1992).

[6] Z. Liliental-Weber, F. Namavar, A. Claverie, Ultramicroscopy 52, 570 (1993). 